\title{
UNA REFLEXIÓN SOBRE LA FORMACIÓN SISTÉMICA COMO CONSTRUCCIÓN INTERSUBJETIVA
}

Milagros Santamaría Rivas ${ }^{\star}$

\section{Introducción}

A lo largo del siglo XX asistimos a una transformación conceptual sobre el conocimiento y la ciencia. Se reconoce la necesidad de superar las concepciones mecanicistas caracterizadas por supuestos de objetividad, cuya lógica formal se basa en ideas tales como:

- El conocimiento es la representación o lógica del mundo real.

- La existencia de verdades singulares universales, descontextualizadas.

- Las explicaciones dadas por modelos axiomáticos, con métodos prescriptivos, cuyo énfasis principal se guía por mediciones cuantitativas.

- Contextos creados en forma artificial y/o controlados con el objetivo de descubrir leyes de carácter universal.

* Maestría en Terapia Familiar Sistémica (Universidad Politécnica Salesiana). Dra. en Psicología Clínica (Universidad Central del Ecuador); Lcda. en Psicología Clínica (Universidad Central de Venezuela), Psicoterapeuta Familiar Municipio de Quito (UMSC); Docente Maestrías en Universidad Politécnica Salesiana y Católica de Guayaquil (Ecuador). 
Lo que subyace a estos supuestos es la existencia de un mundo real como entorno independiente de cualquier organismo.

Dar paso a nuevas visiones implica un salto epistemológico como proceso reflexivo y forma de conocer, con una lógica que dé cabida a las interacciones y aún a las contradicciones de los fenómenos o procesos tanto humanos como inherentes a otros campos.

En palabras de Bateson reconocer los inconvenientes de vivir en un mundo de tipo "o esto o el otro", como una búsqueda de opciones integradoras que permitan reflexiones sobre la práctica científica y sus limitaciones epistémicas en contextos específicos, con formas de conocimiento y prácticas culturales que organizan modos de vida, de pensamiento y acción.

Específicamente en lo relativo a los fenómenos sociales autores vinculados a diversas disciplinas como la sociología, psicología, educación, terapia familiar (entre otras) coinciden en la necesidad de abstraerse de formular juicios de valor en sus explicaciones y concepciones, entonces el pensamiento sistémico surge como un desafío "que arrebata al hombre de ciencia de poder conocer alguna cosa con certeza ateniéndose simplemente a las reglas del juego" (Ludewing, 1998).

Acorde a lo señalado la formación sistémica se propone como un espacio educativo cuyo basamento epistemológico (visión sistémica) se nutre de tradiciones teóricas unidas por un aspecto común: la inexistencia de verdades únicas, lo cuál nos remite a poner acento en las dinámicas relacionales, dirigidas al desarrollo del ser humano, resultando inseparable los aspectos cognitivos y emocionales como elementos constructivos de formas de conocimiento y procesos de cambio dentro de contextos particulares como el educativo que guarda relación con niveles más amplios. 


\section{El contexto de formación sistémica}

Resulta imposible sustraerse del contexto histórico al referirse a la formación sistémica; desde ahí el pasado, no es un conjunto de hechos sueltos; es una construcción en la cual todos somos responsables directos.

Según Mara Selvini, el contexto se entiende como un marco de referencia cuyas características específicas imprimen reglas de comunicación y relación a quienes participan en él; así como una ubicación temática y física (entre otras) cuya singularidad apuntala la búsqueda de objetivos de personas y grupos humanos considerados como sistemas en mutua relación.

En la actualidad el contexto de formación de la maestría en Asesoría, Terapia Familiar e Intervención Sistémica, se vincula a una sociedad en la cual el debate sobre globalización desde los aspectos pragmáticos y/o tecnológicos y los de multiplicidad, desde lo humano, cultural, relacional se plantean como dos caras de la misma moneda: el fenómeno de la posmodernidad.

Desde esta idea, la formación sistémica se constituye en una exploración continua del desarrollo humano, en los distintos niveles implicados de un contexto ecológico: 1) microsistema: personas, familias, grupos; 2) mesosistema: entornos significativos de las personas, familias, grupos; 3 ) exosistema: relación de diversos hechos con la organización social; 4) macrosistema: correspondencia entre forma y contenido de los micro, exo y meso sistema a nivel de la cultura (Bronfenbrenner, 1987). Unida a la exploración se reconoce el requerimiento de comprender el "convivir humano" como un desafío permanente desde donde el conocimiento deja de ser una certeza y como tal la formación alude a lo que podríamos definir como dar forma o construir conoci- 
mientos a partir de diversas distinciones u operaciones de distintos observadores (estudiantes, docentes, familias, personas, grupos).

Dado que cualquier observador es parte activa del sistema, sus descripciones se consideran como actos auto referenciales, en los cuales se pone de manifiesto relaciones entre distintas identidades, siendo el observador a su vez un instrumento de conocimiento, cuyo compromiso consigo mismo y con el entorno, no es explicar la realidad, sino experimentar comunión con el ecosistema en donde evoluciona, "de hecho cuando el compromiso con la propia vida no existe, cualquier conocimiento es inútil” (Bucay,2004).

Acorde con lo expresado por el pensamiento de Barris, Ortiz y otros, la formación sistémica resulta en sí misma la creación de un contexto particular de múltiples aprendizajes interconectados por medio de un proceso participativo, en el que describir, comprender e interpretar fenómenos sociales, implica abrir la puerta a la evolución humana, con una orientación filosófica basada en principios: ecológicos, abarcativos, transgeneracionales, transculturales, en esencia humanistas, privilegiando el ser histórico, biográfico, con respeto a su dignidad humana y libertad de elección.

Por otra parte la formación es un espacio de encuentro de carácter ético relacional, que cuenta con un marco de aceptación a las diferencias y respeto por los otros como seres autónomos, que son parte de un campo fenomenológico específico: los sistemas psicosociales; unidades complejas que cuentan con una organización y estructura que les permite establecer relaciones entre sus elementos (interacciones, transformaciones), además de preservar su identidad. 
Entonces el carácter ético relacional, es un eje transversal que sostiene el proceso de formación, como una recreación permanente de experiencias de influencia recíproca entre los subsistemas participantes, que contienen componentes cognitivos (pensamientos), pragmáticos (formas de hacer) y emocionales (vínculos afectivos), cabría decir un metasistema (forma de leer el mundo) en el que se reconoce una epistemología cuál sustento de la construcción de significados y/o intersubjetividades.

\section{El pensamiento sistémico como referente de formación}

El pensamiento sistémico esbozado como una forma particular de elaboración sobre las construcciones del conocimiento humano, puede entenderse en contraste con el pensamiento analítico-sintético y/o deductivo, de carácter cartesiano que busca respuestas objetivas a sus interrogantes desde una perspectiva racional y dicotómica, verdadero-falso y como tal un aporte al desarrollo tecnológico. Opera en forma inductivo-deductiva, entendido como un complemento dialéctico, un proceso que se genera con la influencia del entorno y la capacidad de cada sistema de elaborar sus propias estructuras. Es cartográfico, busca la descripción de quien describe, es decir una teoría del observador.

El pensamiento sistémico cuestiona las explicaciones lineales para dar paso a la recursión entendida como el reconocimiento de un determinismo recíproco que asume la existencia de sistemas complejos, que necesitan relacionarse con entornos cambiantes y como tal se mueven en la incertidumbre. A su vez estos sistemas cuentan con un orden jerárquico de retroalimentación, una dinámica multifacética que solo puede entenderse 
en conexiones interexplicativas. Podría conceptualizarse como: plural-divergente, en tanto valida las diferencias en las cuales no hay privilegio de un tipo de determinantes sobre los demás; sale de las variantes del mecanicismo causal; descarta el determinismo interno o el ambientalismo extremo. Las explicaciones adquieren una resignificación constante acorde a los contextos; se entiende a los sistemas como conjuntos relacionales en continua movilidad.

El pensamiento sistémico puede entenderse como una síntesis de planteamientos de ciencias naturales, humanistas y sociales en la cuál los sistemas son construcciones de conocimiento humano que surgen como un movimiento circular de observar y pensar ( $\mathrm{Lu}-$ dewing, 1998).

Bradford y Keeney sostienen que la evolución de la práctica sistémica, y como tal de su marco epistemológico, ha pasado por el reconocimiento de que las dualidades aparentes no son tales y por tanto se requiere trazar distinciones entre los subsistemas implicados evitando el establecimiento dicotómico lineal y progresivo entre lo material y lo espiritual o entre mente y cuerpo.

La anterior puede considerarse una de las premisas importantes de la llamada primera cibernética, cuya aplicación operativa se observa en el estudio de la manera en que los procesos de cambio determinan el orden de estabilidad o control en los sistemas, desde una postura del observador externo.

A su vez resulta relevante la contribución de la llamada cibernética de segundo orden según la cual el observador se incluye en el sistema o fenómeno observado reconociendo sus restricciones, por tanto no puede ejercer control, ya que asume que los sistemas están sujetos a órdenes superiores de auto corrección. 
Entonces los procesos sistémicos surgen por principio unos de otros y se pueden considerar como estructuras complementarias que pueden cambiar de manera congruente (Ludewing, 1998).

Conocer es un proceso con determinantes multicausales, según esta noción es importante entender el conocimiento como acción efectiva en donde docentes y estudiantes requieren una ética particular como marco relacional que incluya el respeto por las diferencias y la conciencia de ser partícipe en la configuración del sistema de formación, definido por demandas mutuas.

\section{El pensamiento sistémico maneja la perspectiva fenomenológica}

El pensamiento sistémico entiende los fenómenos sociales desde la perspectiva del actor, examina el modo en que los seres humanos experimentan el mundo. La realidad que importa es la considerada por las personas, las fuerzas que les mueven no son simplemente el cuerpo humano, sino sus ideas, sentimientos, motivos internos, así como la construcción de significados a partir de la influencia de variadas interrelaciones con otros actores.

"El significado viene a ser el resultado de la relación establecida entre los hechos o fenómenos y la corriente interna de la conciencia del actor, donde el fenómeno aparece en el momento cuando el actor reflexiona acerca de sus vivencias"

Rubén Leal, 2007: 8.

Sobre el contexto: desde esta perspectiva, estudiantes y docentes acceden al conocimiento del contexto como interlocutores para lo cuál las vivencias cobran sentido desde la posición que un actor adopta en relación con 
otro, la cual dependerá del tiempo, lugar y la interpretación que se haga, aceptando al otro como alter ego que puede conocer a su interlocutor así como este puede conocerlo, encontrándose en la posibilidad de establecer relaciones horizontales (simétricas) desde la propuesta de la noción de intersubjetividad (Leal, 2007) y complementarias desde el recorrido experiencial del docente así como sus reflexiones teóricas.

El reconocimiento de la complejidad: pasa por tratar de comprender las múltiples relaciones existentes entre los sistemas como propuesta de formación, incluye el reconocimiento de una epistemología cibernética subyacente y por tanto una concepción inclusiva y compleja de dichas relaciones, lo cual lleva a pensar que cualquier separación entre subsistemas, es una acción artificial, ya que el entendimiento viene dado por una operación recursiva, propia de los sistemas._Reconstruir una realidad sistémica implica hacer distinciones y o contar con una teoría sobre las teorías o una descripción de las descripciones, lo cual pone de relieve un enfoque recursivo de la epistemología que aplicado a la práctica puede explicitarse como la visón de metacontexto, contexto y conducta.

Toma como referente los sistemas sociales: dado que los sistemas sociales constituyen parte fundamental de un contexto de formación sistémica, es importante plantearlos como premisa a su finalidad. Desde las ideas sistémicas los planteamientos biológicos y sociológicos aparecen más que como complementarios o diferentes, interrelacionados. Es así como Maturana afirma que el ser individual es necesariamente social y viceversa, el mismo autor explica que los sistemas sociales se basan en el hecho de que los individuos realizan autopoyesis, presuponiendo estructuras orgánicas complementarias que pueden cambiar de forma congruente, indica además que los 
sistemas sociales derivan de un consenso entre los seres humanos, participando de un reciproco acoplamiento estructural y cooperación en el sostenimiento del entramado relacional (Ludwing, 1998).

Luhmann por su parte establece un distinción entre sistema y entorno definiendo a los sistemas sociales por la comunicación, teniendo como consecuencia que las fronteras sistémicas se revelan como fronteras de sentido, siendo este ultimo el marco referencial para abordar conceptualmente estabilidad y cambio de los sistemas incluyendo el de formación y sus efectos recíprocos.

El sentido orienta la formación de enlaces y preserva la complejidad. Los componentes de los sistemas sociales se constituyen como unidades autónomas que surgen en coevolución con los contextos a los que se vinculan. Así los sistemas sociales pueden ser vistos desde la influencia sociológica, biológica y psicológica con explicaciones interdependientes que se conectan en una meta visión de carácter ecológico.

Cabe considerar los anteriores planteamientos como referentes de una formación vista como sistema socio-educativo, en el cuál se destaca la condición proactiva del conocimiento desde la perspectiva de construcción dialéctica, que apunta hacia la generación de significados personales e interpersonales entre los miembros del sistema, a través de un proceso experiencial, explicativo y por ende reflexivo, integrador del campo fenomenológico (relacional descriptivo) y ontológico (significado de ser humano, sentido de sí mismo).

En consonancia con lo expuesto, la formación sistémica propone el conocer humano como un desarrollo inacabado, gradual en cuyo camino se requiere de ajustes continuos a procesos adaptativos (de si mismos y con otros) en un contexto que destaca los componentes intersubjetivos de las experiencias educativo formativas. 
Dado que la formación sistémica se organiza alrededor del desarrollo de los seres humanos es relevante tomar en cuenta la existencia de procesos afectivo-emocionales que apuntan a distintas realidades como interconstrucciones de significado y/o intersubjetividades. Según Humberto Maturana, todo sistema racional tiene una base emocional, la cuál se corresponde con la percepción del mundo de las personas, siendo esta última una apreciación subjetiva, sustentada en la autoimagen, que solo adquiere significado en un contexto relacional, en otras palabras en la conexión con otras subjetividades.

Acorde a lo expuesto el componente intersubjetivo cobra valor en la formación sistémica, como un proceso de saberes, reflexiones y acciones a diferentes niveles, en el cuál subyace la idea de un otro/a como alter ego, estableciéndose un vínculo emocional, creado en la percepción de semejanza con seres significativos (docentes, compañeros, familias) en el desarrollo de aprendizaje, cuyo carácter circular explica a la vez que vuelve responsable del proceso de formación, a todos los involucrados. En lo relativo a la generación de una "realidad" intersubjetiva, mediante la implicación de un otro/a con una historia construida intergeneracional y culturalmente.

Podría pensarse en los miembros del sistema de formación como historias vivientes que para recrearse buscan espacios de seguridad con múltiples interrogantes entre las que se pueden citar: ¿Cómo se define el sistema de formación? ¿Qué roles juegan sus miembros? ¿De qué manera influyen las creencias y situaciones emocionales en el aprendizaje? ¿Qué significa un espacio de seguridad en lo referente a la formación sistémica?

La noción de intersubjetividad resulta un elemento clarificador en la definición del sistema de formación, en los planos teórico y vivencial, como enfoque ontológico de la persona orientado hacia los procesos de sig- 
nificado en los cuales el ordenamiento del entorno que realiza cada sistema, es inseparable de su vinculación con él (Guidano. 1994). Siguiendo esta idea, el rol del docente en la formación sistémica es de un facilitador, cuál constructor de puentes vinculares, influyendo en este proceso, sus experiencias emocionales significativas, entendidas como una revaloración del sentir bases que apoyan el reconocimiento de la emocionalidad de otro/a (estudiante) siendo consecuencia de este proceso intersubjetivo la autoindividuación y experimentación de límites propios.

Acorde a lo expresado, privilegiar lo referente a los vínculos afectivos requiere remitirse a temas de proximidad y distancia emocional en el contexto formativo; al respecto las formas vinculares pueden explicarse conceptualmente a partir de la teoría del apego que se refiere a los diferentes modelos de establecer proximidad afectiva con seres significativos a lo largo del ciclo vital humano.

Se reconoce que el estilo de apego desarrollado en la infancia se expresa en todos los momentos de la vida, también se exhibe en la formación, constituyéndose en sistemas de significados intersubjetivos que organizan la experiencia de aprendizaje dándole forma a posturas, es decir desde donde se mira y concibe a otro/a a partir de los patrones de apego personales. En términos generales estas posturas se evidencian como apego seguro (estar fácilmente disponibles a la solicitud de comprensión y protección de otros/as) y apego ansioso (inconsistencia en las respuestas de búsqueda de acercamiento y protección). Siendo ambos la expresión de una organización vincular, son susceptibles de recrearse en nuevos procesos de evolución relacional marcados por relaciones humanizadas desde similitudes y diferencias en acercamientos afectivos como una de las bases constitutivas de sí mismo, siendo un aporte a la comprensión de la interacción y la cognición cuál síntesis dia- 
léctica cuyo fundamento sostiene en parte el sistema de formación (Greenberg, Rice, Elliot, 1998).

A la consideración de los factores emocionales en la formación sistémica como aspectos básicos subyacen algunas ideas. Las emociones no se almacenan, se reconstruyen o reorganizan, creando nuevos significados a partir de procesos intersubjetivos, es así como un contexto seguro para la autoexploración en un ambiente empático, cálido y de aceptación favorece el proceso de crecimiento en todos los participantes, convirtiéndose en otros significativos, capaces de compartir la sensibilidad en un entorno de respeto.

A su vez anima al docente a buscar lo que es evolutivamente posible, es decir expandir la conciencia (autoconciencia) reforzándose la capacidad de elección y elaboración de acciones que generan diversos estilos a partir de recursos personales, desde el principio de autoorganización de los sistemas humanos como expresión de distinto significado.

Como ya se dijo, formar, "dar forma" es una propuesta y como tal debiera ser lejana a la imposición, cercana al estímulo de ser, hacer y saber con otros, distinguiendo cualidades como la flexibilidad, responsabilidad, curiosidad que entre otras fomentan apertura sin dañar la integridad de otros/as.

Vista así la formación sistémica es un conjunto de respuestas a múltiples demandas cuyo énfasis en el ser, convierte al otro en un espejo donde mirarse y mirar, elaborando un nosotros con similitudes y diferencias expresadas en un ejercicio de aprendizaje en el cuál los vínculos afectivos son tomados como guías en el accionar, a partir de la comprensión de reacciones emocionales como aspectos relevantes que aportan a la solución de problemas y dilemas humanos propios del sistema de formación. 
Para los actores del proceso educativo sistémico el tomar conciencia sobre diferencias y semejanzas en el plano emocional puede conducir hacia variadas formas de afrontamiento y/o reconocimiento de las necesidades humanas asociadas con los sentimientos personales e interpersonales, toda vez que se consideran los afectos como aspectos relacionales orientados por creencias y expectativas (a nivel macro y micro social) de lo que las personas son y deberían ser.

Este intercambio de emocionalidad se hace posible en un contexto que potencia la exploración y el cambio del ser, otorgando para ello herramientas que permiten reconocer y expresar las vivencias intersubjetivas, legitimando el sentido de si mismo desde la confirmación vista como apego seguro (posibilidad de aproximarse en la aceptación) para lo cual resulta clave en el plano operativo desde el rol docente:

- Respetar los ritmos personales

- Legitimar los sentimientos

- Contextualizar los eventos

- Explorar empáticamente

- Facilitar la reflexión crítica del sistema

Según lo planteado, el componente intersubjetivo se constituye en un factor organizador de la formación sistémica, directamente relacionado con el eje del ser como constructor de significados en contextos específicos, cuyo carácter cambiante se enmarca en una dinámica continua de reconstrucción de sí mismo como proceso intersubjetivo. 


\section{Conclusiones}

- La formación sistémica como proceso educativo se identifica con posturas teórico epistemológicas que se mueven entre el constructivismo y el post-racionalismo en tanto:

- Se nutre de diversas teorías para el entendimiento de los fenómenos relacionales, desde una postura crítica permanente.

- Da importancia a las emociones como factores relevantes de cambio en las personas y como tal, niega posturas de neutralidad en el proceso formativo

- Siendo los miembros de un sistema actores de sus procesos, reflejan significados que dan carácter a sus formas relacionales produciéndose un fenómeno recursivo de conexiones a diferentes niveles ecológicos

- Asumir el carácter auto-referencial del conocimiento, resta sentido a la objetividad de preceptos explicativos de carácter lineal, y en su lugar privilegia la visión de múltiples realidades que conviven en amplios contextos.

- Se considera a los vínculos como fenómenos humamos esencialmente afectivos, lo cual se connota sistémicamente como el cúmulo de emociones que afectan al ser relacional en distintos ciclos vitales y de aprendizaje, reconociéndolos también como ecos que resuenan entre personas acercándolas o distanciándolas, así los componentes intersubjetivos aportan a un diálogo de vínculos, que introduce diferencias y semejanzas significativas en lo referente al desarrollo humano.

- Como proceso educativo, la formación sistémica se expresa en relaciones entre sujetos, como tal contradice la lógica mecanicista, reduccionista del aprendizaje, según la cuál el docente se convierte en un programa- 
dor y los estudiantes pasan por los mismos procesos sin reconocer sus intencionalidades (Gallegos, 2007), privilegiando preinscripciones unidireccionales de tipo instrumental que desconocen el significado de las vivencias y por ende de la intersubjetividad.

- Como proceso dialógico, la formación apunta al logro de competencia relacional, entendida como una postura ante la vida que favorece la autonomía, la discrepancia razonada, la originalidad, la productividad en ausencia del dogmatismo.

- Como proceso estratégico, se orienta hacia la organización del significado personal con especificidades individuales e interpersonales, entendiendo que la formación es más un arte que una tecnología, un proceso comprensivo más que predictivo con un sentido de totalidad e interconexión (Gallegos, 2007).

Entonces la formación sistémica puede verse como una metalectura (lectura de muchas lecturas) con importantes diferencias entre armar y construir. Cuando armamos podemos servirnos de un manual que indica paso a paso conductas a seguir. Cuando construimos creamos, diseñamos, soñamos, nos equivocamos, aprendemos y sobretodo nos preguntamos constantemente: qué hacer, cómo hacer, a quiénes servirá lo que hacemos, cómo lo verán, cómo nos sentiremos al ver nuestra obra, qué podemos dar de nosotros, qué se nos dificulta, quién nos puede ayudar y así un sinfín de interrogantes que mantienen vivo el interés por aventurarse a proclamar humanidad con un sentido ético, según el cuál toda persona es un interlocutor válido con distintas posturas que solo se reconoce en el dialogo en la posibilidad de reflexión sobre sí mismo y el impacto que la historia tiene sobre la vida humana (Ortiz, 2008). 


\section{A título de reflexión final}

Ni verdad, ni certeza. Se ellas perjuré

En mi noviciado, tal como los jóvenes.

Deben abjurar del mundo al ser llamados

A las órdenes sagradas.

"Si...entonces"... solo esto afirmo:

$y$ mis éxitos, son como hermosas cadenas

uniendo dudas gemelas, por que es vano pedir

la justificación de lo que postulo,

o el sello de lo concreto, en lo que demuestro.

Sin embargo se levantan puentes.

$Y$ los hombres ya no se arrastran en dos dimensiones.

$Y$ tales triunfos proceden.

No en pequeña medida, del poder de este juego

Jugado con las sombras tres veces atenuadas.

Que las cosas tienen sobre sus originales.

¿Que frágil la varita, pero cuán profundo el hechizo!

Clarence Wylie

\section{Bibliografía}

BALBI, Juan:

2004 La mente Narrativa. Buenos Aires: Paidós.

BARRIS, Abdelganhi. ORTIZ, Dorys y OTROS:

2007 Propuesta MIATEFAS. UPS, Quito

BRADFORD, Keeney:

2001 Estética del Cambio. Buenos Aires: Paidós.

BUCAY, Jorge:

2004 Shimriti. De la ignorancia a la sabiduría. Buenos Aires:

Océano.

GALLEGOS, Ramón:

2007 Integralidad y Calidad Educativa. México:

GREEMBERG, RICE, ELLIOT.

1998 Facilitando el Cambio Emocional. Barcelona: Paidós. 
GUIDANO, Vittorio:

1994 El sí mismo en proceso. Barcelona: Paidós.

LEAL, Rubén:

2006 "La sociología interpretativa de Alfred Schütz. Reflexiones en torno a un planteamiento epistemológico cualitativo", en Alpha 23 (2006):201-212.

LUDEWIG, Kurt:

1998 Terapia Sistémica. Barcelona: Herder

NEIMEYER, Gregg:

1996 Evaluación Constructivista. Barcelona: Paidós

NEIMEYER, Robert:

1998 Constructivismo en Psicoterapia. Barcelona: Paidós.

ORTIZ, Dorys:

2008 La Terapia Familiar Sistémica. Quito: Abya-Yala.

WYLIE, Clarence:

1957101 Rompecabezas en el Pensamiento y Lógica. Inc: Dover Publications. 